SECTION III

\title{
STELLAR AND SOLAR UV RADIATION
}

\section{Chairman:}

L. HOUZIAUX 


\title{
IDENTIFICATIONS OF EMISSION LINES IN THE EUV SOLAR SPECTRUM
}

\author{
CAROLE JORDAN \\ Astrophysics Research Unit, Culham Laboratory, Abingdon, Berks., England
}

\section{Introduction}

In this brief review of solar line identifications the progress made since the previous conference in this series, which was held in Maryland in 1968, will be discussed.

Only lines which appear in emission when seen in the solar disk will be included, thus excluding lines from low stages of ionization at wavelengths of above about $2000 \AA$ which can appear in emission at the limb.

It is convenient to divide the solar spectrum between about $1.5 \AA$ and $2300 \AA$ into wavelength regions, since the various types of transitions which will be discussed tend to appear over restricted wavelength ranges.

There are several papers on solar line identifications in this issue and the reader is referred to these for details where appropriate.

\section{The region $1.5-44 \AA$}

The solar observations of this spectral region have been considerably extended since 1968. The strongest lines observed arise from helium-like and hydrogen-like ions. In 1969, Gabriel and Jordan (1969a) identified a strong line to the long wavelength side of the OVII resonance line, and similar lines close to other members of the iso-electronic sequence, as being due to the forbidden $1 s^{2} S_{0}-1 s 2{ }^{3} S_{1}$ transition. The resonance line, $\left(1 s^{2}{ }^{1} S_{0}-1 s 2 p^{1} P_{1}\right)$, intercombination line, $\left(1 s^{2}{ }^{1} S_{0}-1 s 2 p{ }^{3} P_{1}\right)$, and forbidden line have now been observed from the helium-like ion of all abundant elements between $\mathrm{Cv}$ and Fexxv (Batstone et al., 1970; Doschek and Meekins, 1970; Freeman and Jones, 1970; Meekins et al., 1970; Neupert and Swartz, 1970; Walker and Rugge, 1970; Doschek and Meekins, 1970; Doschek et al., 1971; Grineva et al., 1971; Neupert, 1971). In flare spectra the higher members of the resonance series of hydrogenic and helium-like ions, up to $\Delta n=5$ in most ions, are also observed. Since Doschek is presenting an extensive review including details of these identifications (See the paper by Doschek, this issue, p. 765) they will not be discussed further here.

Gabriel and Jordan (1969a) also classified other groups of lines apparent in laboratory spectra close to the helium-like ion resonance lines, as inner shell transitions between the configurations $1 s^{2} 2 s, 1 s^{2} 2 p$ and $1 s 2 s 2 p, 1 s 2 p^{2}$ in lithium-like ions. Some of these transitions have since been reported in solar spectra from the lithium-like ions of abundant elements above and including Ne vill (Neupert and Swartz, 1970; Doschek et al., 1971; Grineva et al., 1971; Neupert, 1971; Parkinson, 1971; Walker and Rugge, 1971). 
The spectral resolution obtained by Grineva et al. (1971) is sufficient to resolve about a dozen lines between $1.85 \AA$ and $1.87 \AA$, and these authors have proposed classifications for six inner shell transitions in FexxIv.

Walker and Rugge (1971) have observed satellite lines to the hydrogenic ion MgXII resonance line and have proposed classifications for these in terms of lines from doubly excited states in MgXI. Doschek (this issue, p. 765) suggests that similar lines have also been seen in the NRL data for SixIV.

The review papers by Walker (this issue, p. 672) and by Gabriel (this issue, p. 655 ) should be referred to for further details of satellite line identifications and for comparison between theoretical and observed intensities.

The early work of Neupert et al. (1967) and the later observations by Walker and Rugge (1969) have shown that the spectrum between 10 and $20 \AA$ is considerably enhanced during solar flares. The pre-flare spectrum is dominated by $2 p^{6}-2 p^{5} 3 s, 3 d$ transitions in Fexvil (see e.g. Blake et al., 1965). Neupert et al. proposed that the strong transitions in the flare spectra are due to $2 p^{n}-2 p^{n-1} 3 s, 3 d$ transitions in the higher ions FexvIII to Fexxiv, but the limited resolution of the solar spectrum made precise classifications difficult. Later, Feldman and Cohen (1968) listed possible configurations and stages of ionization derived from laboratory work. The recent laboratory work by Fawcett (1971a) has provided detailed classifications for lines from the ions Fe XIX and $\mathrm{xx}$ and from the isoelectronic sequences leading to FexviII and FexxI. Fawcett concluded that further solar spectra of higher resolution are still necessary before the identifications can be completed.

\section{3. $40-170 \AA$}

There have been no recent major developments in identifications proposed for lines in this wavelength region. Freeman and Jones (1970) and Feldman et al. (1972) have recently obtained solar spectra in this regiom. The lines reported are mainly transitions of the type $2 p^{n}-2 p^{n-1} 3 s, 3 d$ and $2 s^{n}-2 s^{n-1} 3 p$ in ions of magnesium, silicon and sulphur or transitions of the type $3 p^{n}-3 p^{n-1} 4 s, 4 d$ and $3 s^{n}-3 s^{n-1} 4 p$ in the ions FeIX-FexvI. About two-thirds of the lines reported have proposed identifications. Many of the identifications in this region were made by Widing and Sandlin (1968), from the earlier NRL spectra, or have derived from the laboratory work of Fawcett et al. (1968).

In the latter paper Fawcett et al. also gave the wavelength regions where transitions of the type $3 p^{n-1} 3 d-3 p^{n-1} 4 p, 4 f$ would be expected. Jordan (1968) suggested that these transitions could account for many of the unidentified lines in the solar spectrum between $80 \AA$ and $170 \AA$. Recently, Wagner and House (1971) have proposed classifications for some twelve $3 p^{5} 3 d-3 p^{5} 4 f$ transitions in laboratory spectra of FeIx. The lack of detailed correlation between the classified laboratory lines and unidentified lines in the spectrum obtained by Feldman et al. is however, disappointing. Further laboratory work on the $3 p^{n-1} 3 d-3 p^{n-1} 4 f$ transitions in other ions would be of value, for, as pointed out by Wagner and House, they can be used to determine the separations of metastable levels in the $3 p^{n-1} 3 d$ configurations, transitions between which can give rise to forbidden transitions in the visible and near UV regions of the solar spectrum. 


\section{4. $170-450 \AA$}

\section{A. $170-240 \AA$}

The laboratory work of Fawcett has continued to provide classifications for transitions of the type $3 p^{n}-3 p^{n-1} 3 d$. From his recent work Fawcett (1971b) has identified a further eight lines of FexIII and one of FexII between $200 \AA$ and $240 \AA$ in the spectrum obtained by Freeman and Jones (1970). Widing et al. (1971, and this issue, p. 665) in a paper presented at this conference propose several of the same transitions and three further Fe XIII identifications from recent NRL spectra. The spectra presented by Feldman et al. at this conference have a higher wavelength resolution and accuracy than any other published spectrum. They have succeeded in resolving several lines previously observed as blends and report many new lines between $180 \AA$ and $240 \AA$. Since the conference Fawcett (private communication) has pointed out that some 23 unidentified lines in the data of Feldman et al. for this wavelength region can be identified as lines of FexI-XIII (Fawcett, 1971b) and of NiXII and XVI (Fawcett and Hayes, 1971). There remain about 40 lines, out of the 130 observed, for which no identifications have been proposed. The majority of these unidentified lines have not been previously observed in the solar spectrum.

\section{B. $240-450 \AA$}

The observed transitions in this region are, apart from the Lyman series of He II, predominantly of the type $2 s^{2} 2 p^{n}-2 s 2 p^{n+1}$, in magnesium, silicon and sulphur, and of the type $3 s^{2} 3 p^{n}-3 s 3 p^{n+1}$ in iron. Earlier work on $2 s^{2} 2 p^{n}-2 s 2 p^{n+1}$ transitions has been extended by Fawcett (1970a) and Fawcett et al. (1971); in particular Fawcett (1971c) has recently made a useful compilation of all available data on transitions of this type, from both his own laboratory work and that of other groups.

In a paper at this conference Fawcett discusses his recent laboratory classifications of $3 s^{2} 3 p^{n}-3 s 3 p^{n+1}$ transitions (Fawcett, 1970b, 1971b), which have enabled him to identify a further nine lines of FeX-XIV in the solar spectrum between 240 and $360 \AA$ obtained by Freeman and Jones (1970). Widing et al. (this issue, p. 665) presents spectroheliograms obtained by NRL and also reports identifications for lines of FexXIV; some of these must be revised in the light of Fawcett's recent laboratory data. However, there are several lines apparent in the NRL data, which in the spectra obtained by Freeman and Jones are masked by higher order lines. A flare is apparent in the NRL data obtained on November 4 1970; not only were lines from Nixvil and Nixvin recorded but also lines from the high members of the lithium isoelectronic sequence, SxIV, ArXVI and CaXviII. The spectrum obtained by Feldman et al. (1972) also covers the range $240-450 \AA$ and records the majority of the iron lines.

An important feature of Fawcett's laboratory work is the classification of several inter-system transitions and lines from a common upper level, which provide the separations of levels in the ground configurations, thus checking identifications of forbidden lines in the near UV and visible regions of the solar spectrum.

It is possible that some of the lines remaining unidentified are due to other inter- 
combination lines in FeIX-XIV. In particular, Svensson and Eckberg (1968) have classified a line observed in their laboratory spectra at $217.10 \AA$ as being due to the transition $2 p^{6}{ }^{1} S_{0}-2 p^{5} 3 d^{3}{ }^{3} D_{1}$ in FeIX. Calculations, by the writer, of the relative intensities of FeIX lines, made using transition probabilities calculated by Wagner and House (1969) and approximate cross-section data, indicate that at laboratory densities $\left(N_{\mathrm{e}} \sim 10^{18} \mathrm{~cm}^{-3}\right)$ the $2 p^{6}{ }^{1} S_{0}-2 p^{5} 3 d^{3} P_{1}$ line should be slightly stronger than the transition from ${ }^{3} D_{1}$, and should therefore also be observable. However at solar densities $\left(N_{\mathrm{e}} \sim 10^{9} \mathrm{~cm}^{-3}\right)$, the ${ }^{1} S_{0}{ }^{3} P_{2}$ line should be the strongest Feix intercombination line, followed by ${ }^{1} S_{0}-{ }^{3} P_{1}$, with ${ }^{1} S_{0}{ }^{3} D_{1}$ an order of magnitude weaker and therefore unlikely to be observed in the solar spectrum. Several groups have reported a solar line at $217.10 \AA$ and Feldman et al. (1972) include the ${ }^{1} S_{0}-{ }^{3} D_{1}$ identification in their recent list, but further work on the FeIX energy levels and excitation cross-sections is needed before this identification can be regarded as certain. (See Figure 1 for the FeIx term scheme.)

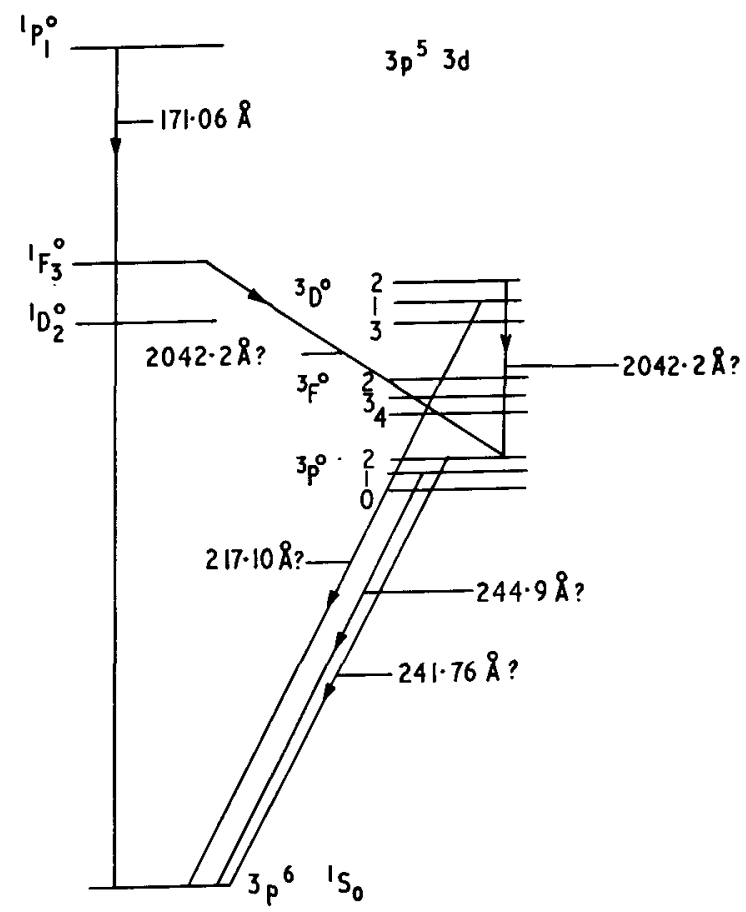

Fig. 1. Schematic term diagram for Fe IX, showing transitions discussed in the text.

The wavelengths calculated by Wagner and House (1971) for the ${ }^{3} D_{1},{ }^{3} P_{2}$ and ${ }^{3} P_{1}$ transitions are $221 \AA, 246 \AA$ and $250 \AA$ respectively. If the laboratory classification of ${ }^{1} S-{ }^{3} D_{1}$ is correct then the other transitions may also occur at wavelengths shorter than those calculated by Wagner and House. One of the strongest unidentified solar 
lines is at $241.7 \AA$ and it is possible that this line is due to ${ }^{1} S_{0^{-}}{ }^{3} P_{2}$. The ${ }^{3} P_{1}$ line would then be expected at $244.9 \AA$ using the ${ }^{3} P_{1}-{ }^{3} P_{2}$ separation calculated by Wagner and House. The line observed by Freeman and Jones at this wavelength is listed as CIV, but this identification is by no means satisfactory because of the intensity of the line and it is possible that the line is due to FeIX ${ }^{1} S_{0}-{ }^{3} P_{1}$. However, the ${ }^{3} D_{1}$ and ${ }^{3} P_{2}$, ${ }^{3} P_{1}$ identifications are mutually exclusive if the coronal line at $2042.2 \AA$ is due to Fe IX. (See Section 6.)

The line observed at $\simeq 417 \AA$ has been identified for some years as the intercombination line $3 s^{2}{ }^{1} S_{0}-3 s 3 p{ }^{3} P_{1}$ in Fexv (e.g. Hall et al., 1963). However there has been some discussion in the literature of whether or not this identification is correct. Bely and Blaha (1968) found that the calculated intensity of the intercombination line, relative to that of the resonance line at $284 \AA$, was more than an order of magnitude less than that observed.

In view of this Hall and Hinteregger (1970) tentatively suggested that the line at $\simeq 417 \AA$ might be due to the lithium-like SxIv transition. However, Fawcett's (1970a) recent laboratory wavelengths for the SxIv lines and the higher wavelength resolution of Hall and Hinteregger's more recent data have ruled out this possibility. Further, their observations of the intensity ratio, (also from Hall, private communication, 1971) give lower values than the earlier estimates. Flower (1971) has recalculated the collision strengths for Fexv transitions; using these and the more recent observations, Flower and Jordan (1972) have discussed the whole problem of the identification of the line at $\simeq 417 \AA$, and conclude that it is due to Fexv, as originally proposed, and that under quiet sun conditions the SXIV line would have an intensity of less than one third of the Fexv line. Widing et al. (this issue, p. 665) reports that in the NRL data obtained during a flare, both lines are apparent.

\section{5. $450-1100 \AA$}

As this region of the spectrum has been observed for many years there have been no recent major line identification problems. The facility of pointing above the limb, used by the Harvard College Observatory group on OSOIV and by the Astrophysics Research Unit on stabilized Skylark rockets, has led to identifications of lines which are weak in spectra from the centre of the disk. The increased path length at the limb enhances the intensities of optically thin lines relative to those of many of the strong resonance lines which become optically thick at the limb. In particular, Dupree and Reeves (1971), making use of unpublished work by Munro (1971), report the resonance lines from the lithium-like and beryllium-like ions of two elements with low abundance, viz. sodium and aluminium.

The observations of limb spectra, made by Burton et al. (1967), from which several intercombination lines, particularly in oxygen ions, were identified for the first time, have been extended. In a recent paper Burton and Ridgeley (1970) summarize the previous work, and discuss further identifications of intercombination lines. In this wavelength region they report the Si Xı $2 s^{2}{ }^{1} S_{0}-2 s 2 p_{1}{ }^{3} P$ line at $580.85 \AA$. 


\section{6. $1100-2200 \AA$}

Burton and Ridgeley have also identified intercombination lines from SIII, (1696.7 $\AA$ and $1683.5 \AA$ ), from SIV (1416.9 $\AA$ and 1406.0 $\AA$ ), from Sv (1204.3 $\AA$ ) and at longer wavelengths, from CII ( $2328 \AA$ and $2324 \AA$ ). The earlier intercombination line identifications have now been confirmed by the laboratory work of Edlén et al. (1969). Since Burton and Ridgeley obtain both limb and disk spectra, the limb to disk enhancements can be used as a guide to the stage of ionization producing an observed line. They report four lines of apparent coronal origin; two are the FexII forbidden lines identified in their earlier paper. These lines and the other two at $1446 \AA$ and $1467 \AA$ have been confirmed as coronal lines by the 1970 eclipse spectra (Speer et al., 1970; Gabriel et al., 1971) discussed below. However their tentative assignment of FexI ${ }^{3} P_{1}-{ }^{1} S_{0}$ to the line at $1446 \AA$ must be replaced by SivilI ${ }^{4} S_{3 / 2}{ }^{2} D_{3 / 2}$. The line at $1410.9 \AA$ reported as a limb-enhanced transition region line is probably the same line as that at $1409.1 \AA$ reported as a coronal line in the eclipse spectra.

The most recent problem of solar line identifications has occurred in this region of the spectrum. During the 1970 March 7, 1970 eclipse, spectra were obtained by flying a rocket into the eclipse path (Speer et al., 1970). The spectra showed the existence of twenty-eight coronal lines only five of which had been reported earlier (see above). The spectra obtained and the identifications proposed for twenty-one of the lines have been discussed by Gabriel et al. (1971) and in more detail by Jordan (1971). At the time when the majority of the identifications were made, accurate wavelengths were available for only a very few of the coronal lines expected between 1000 and $2300 \AA$. Hence other methods were used in addition to considering wavelengths and wavelength differences. The absolute and relative intensities of possible transitions in abundant elements were calculated and compared with the approximate observed intensities then available. The observed spatial distribution of the emission in each line also proved to be of great value in identifying the lines. Variations of coronal temperature with latitude and the structure of large active regions on the limb made it possible to establish the temperature at which unidentified lines are formed. These variations are apparent in the spectra shown in Figure 2. Table I gives a list of lines for which identifications have been proposed. The temperature at which each line would show maximum emission in a uniform atmosphere is also given. The majority of the lines are due to forbidden transitions between levels in the ground configurations $2 p^{n}$ and $3 p^{n}$.

In the $\mathrm{C}_{I}$ isoelectronic sequence $\left(2 p^{2}\right)$ lines from the ions MgVII, Si IX and SXI are observed. The MgVII line (and also those of AlvII and Sivil) is observed only in one part of the active region where not only is the temperature low but also the density is higher than average. The Si Ix lines and the SxI lines originate from common upper levels $\left({ }^{1} D_{2}\right)$ so that the wavenumber difference $\left({ }^{3} P_{1}-{ }^{3} P_{2}\right)$ is also available as a check on the identifications. The SxI lines appear only in the regions of enhanced activity.

The $\mathrm{N}_{\mathrm{I}}$ isoelectronic sequence $\left(2 p^{3}\right)$ is represented by lines of AlvII and Si vilI. In these ions only the ${ }^{4} S_{3 / 2}-{ }^{2} D_{3 / 2}$ component is observed because of the low A-value 

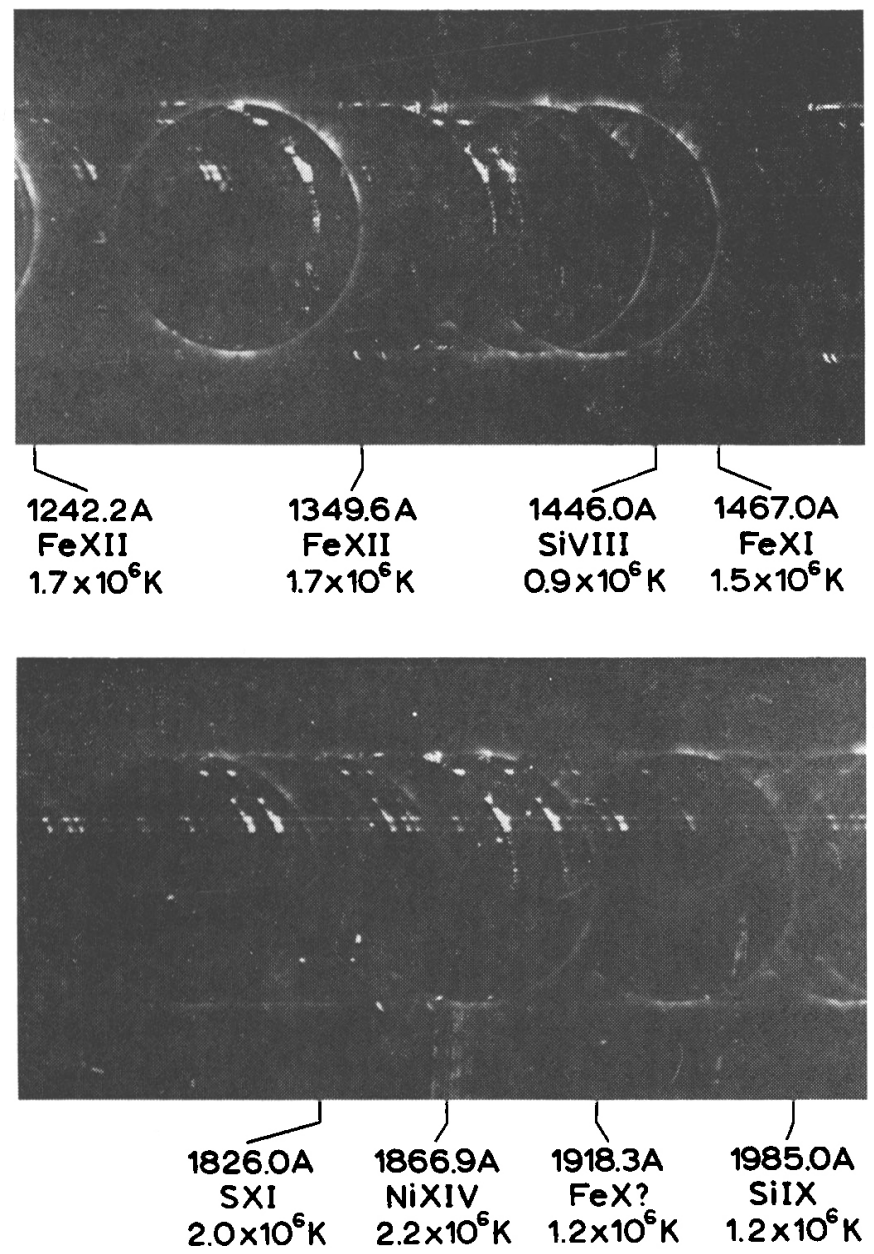

Fig. 2. Enlargement of eclipse frame 30 , showing spatial variation of intensities of lines formed at different temperatures.

for the ${ }^{4} S_{3 / 2}{ }^{2} D_{5 / 2}$ transition. Edlén (1971) predicts the $\mathrm{Sx}\left({ }^{4} S{ }^{2}{ }^{2} D_{3 / 2,5 / 2}\right)$ lines to lie at $1213 \AA$ and $1197 \AA$. The line at $1213 \AA$ is too strong to be $\mathrm{Sx}$ and is identified as FexiII. In $\mathrm{Sx}$ the ${ }^{4} S_{3 / 2}{ }^{2} D_{5 / 2}$ line is expected to be about one third as intense as the line from ${ }^{2} D_{3 / 2}$. However, the spatial distribution of the faint line at $1197 \AA$ is consistent with a line formed at $T_{\mathrm{e}} \simeq 1.5 \times 10^{6} \mathrm{~K}$, lower than that appropriate for a Sx line $\left(T_{\mathrm{e}} \simeq 1.7 \times 10^{6} \mathrm{~K}\right)$.

Lines from Si VII and SIX are observed in the OI $\left(2 p^{4}\right)$ isoelectronic sequence.

The SI $\left(3 p^{4}\right)$ isoelectronic sequence is represented by lines of FeXI and NixIII. The ${ }^{3} P_{1}-{ }^{1} S_{0}$ transition in FeXI is identified with the line at $1467.0 \AA$ rather than the line at $1446.0 \AA$ as suggested earlier by Burton et al. (1967). The NixiII $\left({ }^{3} P_{2}-{ }^{1} D_{2}\right)$ transition is of interest as its wavelength $(2126.0 \pm 0.5 \AA)$ is inconsistent with both of the 
TABLE I

Coronal lines in the EUV spectrum

\begin{tabular}{|c|c|c|c|}
\hline $\begin{array}{l}\lambda(o b s) \\
\AA\end{array}$ & Ion & Transition & $\begin{array}{l}T_{\mathrm{e}} \\
10^{6} \mathrm{~K}\end{array}$ \\
\hline 1190.2 & $\mathrm{Mg}_{\mathrm{VII}}$ & ${ }^{3} \mathbf{P}_{1}-{ }^{1} \mathbf{S}_{\mathbf{0}}$ & 0.69 \\
\hline 1213.0 & FexiIr & ${ }^{3} \mathrm{P}_{\mathbf{1}} \quad{ }^{-1} \mathrm{~S}_{0}$ & 1.85 \\
\hline 1242.2 & FexII & ${ }^{4} \mathbf{S}_{3 / \mathbf{Z}^{-}}{ }^{2} \mathbf{P}_{3 / 2}$ & 1.66 \\
\hline 1349.6 & FexII & ${ }^{4} \mathbf{S}_{3 / 2}-{ }^{2} P_{1 / 2}$ & 1.66 \\
\hline 1446.0 & SiviII & ${ }^{4} \mathbf{S}_{\mathbf{3} / 2}-^{2} \mathbf{D}_{\mathbf{3} / \mathbf{2}}$ & 0.93 \\
\hline 1467.0 & FexI & ${ }^{3} \mathbf{P}_{1}-{ }^{1} \mathbf{S}_{0}$ & 1.45 \\
\hline 1603.2 & Alvil? & ${ }^{4} \mathbf{S}_{3 / 2}{ }^{-2} \mathbf{D}_{3 / 2}$ & 0.7 \\
\hline 1614.6 & $S \times I$ & ${ }^{3} P_{1}-{ }^{1} D_{2}$ & 2.0 \\
\hline 1624.0 & OVII? & ${ }^{3} S_{1} \quad-{ }^{3} P_{2}$ & 2.0 \\
\hline 1715.3 & SIX & ${ }^{3} \mathbf{P}_{2}-{ }^{1} \mathrm{D}_{2}$ & 1.2 \\
\hline 1826.0 & $\mathrm{SXI}_{\mathrm{I}}$ & ${ }^{3} P_{2}-{ }^{1} D_{2}$ & 2.0 \\
\hline 1866.9 & NixIy & ${ }^{4} \mathrm{~S}_{3 / 2^{-}}{ }^{-2} \mathrm{D}_{5 / 2}$ & 2.24 \\
\hline 1918.3 & $\mathrm{Fex}$ ? & $?$ & 1.2 \\
\hline 1985.0 & Silx & ${ }^{3} \mathbf{P}_{1}-{ }^{\mathbf{I}} \mathbf{D}_{2}$ & 1.20 \\
\hline $2042.2^{\mathrm{a}}$ & Ferx & 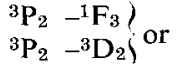 & 0.9 \\
\hline 2085.7 & Nixy & ${ }^{3} \mathbf{P}_{1}-{ }^{1} \mathbf{D}_{2}$ & 2.5 \\
\hline 2126.0 & NixIII & ${ }^{3} \mathbf{P}_{1}-{ }^{1} \mathbf{D}_{2}$ & 2.0 \\
\hline 2147.4 & Sivit & ${ }^{3} \mathbf{P}_{1}-{ }^{1} \mathbf{D}_{2}$ & 0.69 \\
\hline 2149.5 & SilX & ${ }^{3} \mathbf{P}_{2}-{ }^{1} \mathbf{D}_{2}$ & 1.2 \\
\hline 2169.7 & FexiI & ${ }^{4} \mathbf{S}_{3 / 2}-{ }^{2} D_{5 / 2}$ & 1.66 \\
\hline 2185.1 & NixIV & ${ }^{4} S_{3 / 2}-{ }^{2} D_{3 / 2}$ & 2.24 \\
\hline
\end{tabular}

${ }^{a}$ Air wavelengths above $2000 \AA$.

identifications of NixII lines in the visible region being correct. Taking the identification of the ${ }^{3} P_{2}-{ }^{3} P_{1}$ transition at $5115.8 \AA$ as correct, the predicted wavelength for the ${ }^{3} P_{1}-{ }^{1} D_{2}$ transition is $3638 \pm 1.5 \AA$, which does not agree with the line observed at $3642.7 \AA$.

In the $\mathrm{PI}_{\mathrm{I}}$ isoelectronic sequence $\left(3 p^{3}\right)$ lines of FexII and NixIV are observed. The FeXII ${ }^{4} S_{3 / 2}-{ }^{2} P_{1 / 2},{ }^{2} P_{3 / 2}$ lines were identified by Burton et al. (1967) from limb spectra. The FexII ${ }^{4} S_{3 / 2}-{ }^{2} D_{5 / 2}$ transition is very strong in the eclipse spectra, but the transition from ${ }^{2} D_{3 / 2}$ lies at longer wavelengths than were recorded in this experiment. However both the NixIV ${ }^{4} S_{3 / 2}{ }^{2} D_{3 / 2,5 / 2}$ transitions are observed. There are sufficient lines observed to improve the wavelengths predicted for the ${ }^{2} D-{ }^{2} P$ transitions in FeXII. In particular the ${ }^{2} D_{3 / 2}{ }^{2} P_{1 / 2}$ transition can be identified with a line listed by Jefferies (1969) at $3072 \AA$, rather than with the line at $3021 \AA$ as often suggested in the literature.

Lines from FexIII and Nixv are observed from the SiI isoelectronic sequence $\left(3 p^{2}\right)$. The Fexir line is observed at $1213.0 \AA$, close to $\mathrm{H} \mathrm{Ly}-\alpha$. Since the majority of the solar lines were identified, Svensson (1971) and Edlén (1972) have made improved extrapolations for forbidden lines in $2 p^{n}$ and $3 p^{n}$ ground configurations. Their values support all the identifications proposed with the exception of the FexIII ${ }^{3} P_{1}-{ }^{1} S_{0}$ 
transition, for which Svensson obtains 1217.0 $\AA$. However the value of $1213.0 \AA$ has been confirmed by the laboratory work of Fawcett (1971b) in the region 170-400 $\AA$. The Nixv identification was proposed by Edlén (1970, private communication).

The FexI and FexII identifications are also supported by the work of Fawcett (1971b) who has obtained the separations of levels in the ground configurations from his work at shorter wavelengths.

With the exception of the $\mathrm{S} x$ lines, all the transitions expected in the region $1000-$ $2200 \AA$, from abundant elements and suitable stages of ionization, which arise from transitions between levels in either $2 p^{n}$ and $3 p^{n}$ ground configurations, have been observed. The remaining lines must therefore originate from transitions between levels in excited configurations. Three identifications are proposed for transitions between excited levels, although all are tentative. The line at $1624.0 \AA$ may be due to OvII $\left(1 s 2 s^{3} S_{1}-1 s 2 p^{3} P_{2}\right.$ ) but the wavelength measured does not agree with the recent laboratory measurement of $1623.6 \AA$, by Engelhardt and Sommer (1971).

The lines at $2042 \AA$ and $1918 \AA$ are proposed as transitions between levels in the $3 p^{5} 3 d$ and $3 p^{4} 3 d$ configurations in FeIX and $\mathrm{FeX}$ respectively. At present it is not possible to assign terms to the Fex transition. However, the FeIx population calculations discussed earlier and the energy level calculations made by Wagner and House (1971) and Cowan (1965, private communication) can be used to investigate which transitions are likely to be observed. On the basis of Cowan's (1965) calculated energy levels the transition closest in wavelength to that observed is ${ }^{3} P_{2}-{ }^{1} F_{3}$ (Jordan, 1972). However, using the energy levels computed by Wagner and House and the writer's recent calculations of FeIX populations the transition ${ }^{3} P_{2}-{ }^{3} D_{2}$ seems more likely. The writer considers that further laboratory work at short wavelengths is necessary to establish the position of the $3 p^{5} 3 d$ levels, since although the calculations by Wagner and House give a separation which fits with the observed line, their energy for the ${ }^{3} D_{1}$ level does not fit Svensson and Eckberg's observation. Cross-sections for transitions between levels in the $3 p^{6}, 3 p^{5} 3 d$ configurations and also from $3 p^{6}$ to the $3 p^{5} 4 s$, $4 p, 4 d, 4 f$ configurations are needed for the observed intensities of lines to be of use in making identifications.

\section{Conclusions}

During the past three years there have been significant extensions of the solar data available. Over most of the solar spectrum between $1 \AA-2200 \AA$ the new or improved observations have led to interesting problems in line identifications. The identifications have in turn led to new methods of determining the physical conditions in the solar atmosphere, eg electron density determinations from the He I like ion intercombination line to forbidden. line ratio (Gabriel and Jordan, 1969b). The majority of the strong lines have now been identified, either by theoretical considerations or from the extensive laboratory data which have recently become available. However, weak lines may also aid the understanding of the chromosphere and corona and work on the identifications of all remaining features observed must continue. 


\section{Acknowledgements}

I am grateful for preprints received from several authors of papers given at this conference. In particular I wish to thank A. H. Gabriel and B. C. Fawcett for their helpful comments.

\section{References}

Batstone, R. M., Evans, K., Parkinson, J. H., and Pounds, K. A.: 1970, Solar Phys. 13, 389.

Bely, O. and Blaha, M.: 1968, Solar Phys. 3, 563.

Blake, R. L., Chubb, T. H., Friedman, H., and Unzicker, A. E., 1965, Astrophys. J. $142,1$.

Burton, W. M. and Ridgeley, A.: 1970, Solar Phys. 14, 3.

Burton, W. M., Ridgeley, A., and Wilson, R., 1967, Monthly Notices Roy. Astron. Soc. $135,207$.

Doschek, G. A. and Meekins, J. F.: 1970, Solar Phys. 13, 220.

Doschek, G. A., Meekins, J. F., Kreplin, R. W., Chubb, T. A., and Friedman, H.: 1971, Astrophys. J. 170, 573.

Dupree, A. K. and Reeves, E. M.: 1971, Astrophys. J. 165, 599.

Edlén, B.: 1972, Solar Phys., in press.

Edlén, B., Palenius, H. P., Bockasten, K., Hallin, R., and Bromander, J.: 1969, Solar Phys. 9, 432.

Engelhardt, W. and Sommer, J.: 1971, Astrophys. J. 167, 201.

Fawcett, B. C.: 1970a, J. Phys. B. 3, 1152.

Fawcett, B. C.: 1970b, J. Phys. B. 3, 1732.

Fawcett, B. C.: 1971a, J. Phys. B. 4, 981.

Fawcett, B. C.: 1971b, J. Phys. B. 4, 1577.

Fawcett, B. C.: 1971c, Astrophysics Research Unit Report-R2., H. M. Stationery Office.

Fawcett, B. C. and Hayes, R. W.: 1971, J. Phys. B., in press.

Fawcett, B. C., Peacock, N. J. and Cowan, R. D.: 1968, J. Phys. B. 1, 295.

Fawcett, B. C., Gabriel, A. H., and Paget, T. W.: 1971, J. Phys. B. 4, 986.

Feldman, U. and Cohen, L.: 1968, Astrophys. J. 151, 55.

Feldman, U., Behring, W., and Cohen, L.: 1972, this issue, p. 608.

Flower, D. R.: 1971, J. Phys. B. 4, 697.

Flower, D. R. and Jordan, C.: 1971, Astron. Astrophys. 14, 473.

Freeman, F. F. and Jones, B. B.: 1970, Solar Phys. 15, 288.

Gabriel, A. H. and Jordan, C.: 1969a, Nature 221, 947.

Gabriel, A. H. and Jordan, C.: 1969b, Monthly Notices Roy. Astron. Soc. 145, 241.

Gabriel, A. H., Garton, W. R. S., Goldberg, L., Jones, T. J. L., Jordan, C., Morgan, F. J., Nicholls, R. W., Parkinson, W. H., Paxton, H. J. B., Reeves, E. M., Shenton, D. B., Speer, R. J., and Wilson, R.: 1971, Astrophys. J. 169, 595.

Grineva, Yu. I., Karev, V. I., Korneev, V. V., Krutov, V. V., Mandel'stam, S. L., Vainstein, L. A., Vasiljev, B. N. and Zitnik, I. A.: 1971, paper presented at COSPAR, Seattle and to be published in Kosmich. Issled. USSR.

Hall, L. A. and Hinteregger, H. E.: 1970, J. Geophys. Res. 75, 6959.

Hall, L. A., Damon, K. R., and Hinteregger, H. E.: 1963, Space Res. 3, 745.

Jefferies, J. T.: 1969, Mem. Soc. Roy. Sci. Liege, 17, 213.

Jordan, C.: 1968, J. Phys. B. 1, 1004.

Jordan, C.: 1971, Solar Phys. 21, 381.

Meekins, J. F., Doschek, G. A., Friedman, H., Chubb, T. A., and Kreplin, R. W.: 1970, Solar Phys. 13, 198.

Neupert, W. M.: 1971, Solar Phys. 18, 474.

Neupert, W. M. and Swartz, M.: 1970, Astrophys. J. 160, L189.

Neupert, W. M., Gates, W., Swartz, M., and Young, R., 1967, Astrophys. J. 149, 79.

Parkinson, J. H.: 1971, Nature, (Physical Science) 233, 44.

Speer, R. J., Garton, W. R. S., Goldberg, L., Parkinson, W. H., Reeves, E. M., Morgan, J. F., Nicholls, R. W., Jones, T. J. L., Paxton, H. J. B., Shenton, D. B., and Wilson, R.: 1970, Nature 226, 249.

Svensson, L. A.: 1971, Solar Phys. 18, 232.

Svensson, L. A. and Eckberg, J. O.: 1968, Arkiv. Fysik 37, 65. 
Wagner, W. J. and House, L. L.: 1969, Astrophys. J. 155, 677.

Wagner, W. J. and House, L. L.: 1971, Astrophys. J. 166, 683.

Walker, A. B. C. and Rugge, H. R.: 1970, Astron. Astrophys. 5, 4.

Walker, A. B. C. and Rugge, H. R.: 1971, Astrophys. J. 164, 181.

Widing, K. G. and Sandlin, G. D.: 1968, Astrophys. J. 152, 545.

Widing, K. G., Sandlin, G. D., and Cowan, R.: 1971, Astrophys. J. 169, 405.

\section{DISCUSSION}

B. Edlén: (Professor B. Edlén described a recent study of the $Z$-dependence of the level intervals in the configurations $2 s^{2} 2 p^{2}, 2 s^{2} 2 p^{3}$ and $2 s^{2} 2 p^{4}$, relevant to the identification of coronal forbidden lines. In this study, semi-empirical expressions, with a maxium of five parameters, had been fitted to reproduce within experimental error limits all at present available data on these intervals obtained from laboratory and astrophysical observations. The formulae were briefly described and the final results were given in the form of tables of relative level values for the ground configurations of CI-Caxv, Ni-Scxv, and OI-CrXvir.)

B. S. Fraenkel: If the famous line at $217 \AA$ is a ${ }^{1} S_{0}-{ }^{3} \mathrm{D}_{1}$ transition, what is its relative intensity to the ${ }^{1} S_{0^{-}}{ }^{1} P_{1}$ transition?

C. Jordan: The calculations of level populations for a density of $10^{18} \mathrm{~cm}^{-3}$ suggest that the ratio of this line to the ${ }^{1} S_{0^{-}}{ }^{1} P_{1}$ resonance line should be less than $10^{-2}$. I do not know the value of the observed intensity ratio.

H. Nussbaumer: You mentioned discrepancies of a factor 2 for calculated and observed intensities of Fexv $\lambda$ 417. Discrepancies of that magnitude can certainly be attributed to uncertainties in atomic data.

C. Jordan: I agree that a factor of two is within the combined possible errors in the atomic data and measured intensities. 